\title{
Achievable Bisimilar Behaviour of Abstract State Systems
}

\author{
Giordano Pola, Arjan J. van der Schaft and Maria D. Di Benedetto
}

\begin{abstract}
Given a plant system and a desired system, we study conditions for which there exists a controller that interconnected with the plant, yields a system that is bisimilar to the desired system. Some sufficient and some necessary conditions are provided in the general case of (non-deterministic) abstract state systems and stronger results are obtained for the special classes of autonomous abstract state systems, finite abstract state systems, and non-deterministic linear dynamical systems.
\end{abstract}

\section{INTRODUCTION}

A basic question in systems and control theory is to characterize the class of all achievable systems that result from the interconnection of a given plant and a to-be-designed controller. This problem has been extensively studied in the past years in the case of external behaviour equivalence. In fact two main research branches have been developed in the literature depending on the control scheme considered. For example, if we suppose that the controller takes as inputs the state of the plant and an auxiliary control variable, we deal with the classical model matching problem (e.g. [3], [4]). In [3] the model matching problem is defined and characterized for deterministic linear and non-linear control systems while [4] extends these last results to the context of discrete event systems. On the other hand, if the controller does not have access to all the control variables and has as inputs only some components of the output function, i.e. the inputs and outputs of the plant are partially accessible by the controller, the so-called achievable behaviour problem is set (e.g. [19], [22], [5]). In [22], [5] a complete analysis of the achievable behaviour problem is developed for the special class of differential linear systems and [19] extends the results of [22] to the context of general systems.

Different notions of equivalence between dynamical systems have been proposed in the literature and in particular one of the most popular ones in the computer science community is the notion of equivalence by bisimulation, introduced in the 80's of the last century by Milner [7] and Park [9] in the context of concurrent processes. For the classes of non-deterministic concurrent processes [2] and linear systems [21], it was shown that equivalence

This work has been partially supported by the HYCON Network of Excellence, contract number FP6-IST-511368.

G. Pola and M. D. Di Benedetto are with the Department of Electrical Engineering, Center of Excellence DEWS, University of L'Aquila, Poggio di Roio, 67040 L'Aquila, Italy pola, dibenedee ing . univaq. it

A. J. van der Schaft is with the Department of Applied Mathematics, University of Twente P.O. Box 217,7500 AE Enschede, The Netherlands a.j.vanderschaft@math. utwente.nl and with the Institute for Mathematics and Computer Science, University of Groningen, P.O. Box 800, 9700AV Groningen, The Netherlands A.J.van.der.Schaftemath.rug.nl by bisimulation implies external-behaviour equivalence and that the converse implication is true if the systems are deterministic.

Aim of this paper is to consider the problem of achievable behaviour with focus on the bisimulation equivalence rather than on the external behaviour equivalence: we refer to this problem in the following as the achievable bisimilar behaviour problem. The class of systems we consider is that of non-deterministic abstract state systems. This class generalizes the models of [15], [16] to a non-deterministic setting. The proposed model includes as special cases linear and non-linear dynamical systems, Kripke formulas [2], hybrid systems [17], as well as infinite dimensional systems. Given the generality of the model that we consider, the results of this paper are developed in a pure set theory point of view. In particular, some sufficient and necessary conditions are derived for solving the achievable bisimilar behaviour problem. Furthermore a characterization of the class of controllers solving that problem is derived. Finally, by specializing those results to the context of autonomous abstract state systems, finite abstract state systems and non-deterministic finite-dimensional linear systems, we give conditions that are necessary and sufficient for solving the achievable bisimilar behaviour problem. A full version of this paper can be found in [11].

This paper is organized as follows. Section II formally defines abstract state systems and formulates the problem. Section III introduces some new results on bisimulation, simulation and interconnection of abstract state systems that are instrumental for the next developments. In Section IV we characterize the achievable bisimilar behaviour problem for the class of abstract state systems. Section V specializes those results to the case of autonomous abstract state systems, finite abstract state systems and non-deterministic linear dynamical systems. Finally, Section VI offers some concluding remarks.

\section{PREliminaries AND PROBlem STATEMENT}

Aim of this section is to introduce the preliminary definitions and the problem setting of this paper.

\section{A. Abstract state systems and their interconnection}

This section is devoted to the definition of an abstract notion of non-deterministic dynamical systems.

We recall that given a set $\Omega, \prec_{\Omega}$ is a partial order on $\Omega$ if it satisfies the following conditions: (reflexivity) $\omega \prec \Omega \omega$, $\forall \omega \in \Omega$; (transitivity) $\left(\omega_{1} \prec_{\Omega} \omega_{2}, \omega_{2} \prec_{\Omega} \omega_{3}\right) \Rightarrow \omega_{1} \prec_{\Omega} \omega_{3}$, $\forall \omega_{1}, \omega_{2}, \omega_{3} \in \Omega$; (antisymmetry) ( $\left.\omega_{1} \prec \Omega \omega_{2}, \omega_{2} \prec \Omega \omega_{1}\right)$ $\Rightarrow \omega_{1}=\omega_{2}, \forall \omega_{1}, \omega_{2} \in \Omega$. A set $\Omega$ is a partial ordered set 
(poset) if there exists a partial order $\prec_{\Omega}$ on it. A total ordered set $\Omega$ is a poset such that $\forall \omega_{1}, \omega_{2} \in \Omega$, either $\omega_{1} \prec \Omega \omega_{2}$ or $\omega_{2} \prec \Omega \omega_{1}$. Denote by $T$ a poset that represents the time set. Throughout the paper we assume that all the systems involved have the same time set coinciding with $T$. We now introduce the notion of time path that is, roughly speaking, a 'trajectory' embedded in $T$ representing the set of all times 'visited' during an evolution of the system. A time path is a subset $\tau$ of $T$ such that: (ordering condition) $\tau$ is a totally ordered set; (existence of the initial time) $\exists t_{0} \in \tau$ such that $\forall t \in \tau, t_{0} \prec_{T} t$; (continuity condition) $\forall t_{1}, t_{2} \in \tau$ and $\forall t \in T,\left(t_{1} \prec_{T} t, t \prec_{T} t_{2}\right) \Longrightarrow t \in \tau$. Denote by $[T]$ the set of all time paths in $T$. Given $\tau \in[T]$, denote by $t_{0}^{\tau}$ the minimum element in $\tau$ w.r.t. $\prec_{T}$, i.e. $t_{0}^{\tau} \prec_{T} t, \forall t \in \tau$; moreover for any $t^{\prime} \in \tau$, let be $\downarrow_{\tau}^{t^{\prime}}:=\left\{t \in \tau: t \prec_{T} t^{\prime}\right\}$ and $\uparrow_{t^{\prime}}^{\tau}:=\left\{t \in \tau: t^{\prime} \prec_{T} t\right\}$; note that $\downarrow_{\tau}^{t^{\prime}}, \uparrow_{t^{\prime}}^{\tau} \in[T]$. The introduction of time paths is motivated by the necessity to develop a general framework where for example hybrid systems appear as special cases: in fact the notion of time path generalizes the usual notion of hybrid time basis [17], which applies to hybrid systems. Given a set $X$, a pair of time paths $\tau_{1}, \tau_{2} \in[T]$, such that $\tau_{1} \cup \tau_{2} \in[T]$ and $\tau_{1} \cap \tau_{2}=\left\{t^{\prime}\right\}$, and a pair of functions $\chi_{1}: \tau_{1} \rightarrow X, \chi_{2}: \tau_{2} \rightarrow X$, denote by $\left(\chi_{1} \cdot \chi_{2}\right): \tau_{1} \cup \tau_{2} \rightarrow X$ the concatenation of $\chi_{1}($.$) and$ $\chi_{2}($.$) such that \left(\chi_{1} \cdot \chi_{2}\right)(t)=\chi_{1}(t)$, if $t \in \tau_{1} \backslash\left\{t^{\prime}\right\}$ and $\left(\chi_{1} \cdot \chi_{2}\right)(t)=\chi_{2}(t)$, if $t \in \tau_{2}$. We can now propose the following definition of abstract state systems that generalizes the notion of deterministic dynamical system of [15], [16].

Definition 1: An abstract state system $\Sigma$ is a tuple $(X, W, \mathcal{X}, w)$ where:

- $X$ is the state space;

- $W$ is the manifest variables space;

- $\mathcal{X}: X \times[T] \rightarrow 2^{\{\chi:[T] \rightarrow X\}}$ associates to any initial condition $x_{0} \in X$ and any time path $\tau \in[T]$, the set $\mathcal{X}\left(x_{0}, \tau\right)$ of solutions $\chi: \tau \rightarrow X$, such that:

$\circ$ (identity) $\forall x_{0} \in X, \forall \tau \in[T], \forall \chi(.) \in \mathcal{X}\left(x_{0}, \tau\right)$, $\chi\left(t_{0}^{\tau}\right)=x_{0}$

- (semigroup property)

$-\forall x_{0} \in X, \forall \tau \in[T], \forall \chi(.) \in \mathcal{X}\left(x_{0}, \tau\right), \forall t^{\prime} \in \tau$, $\exists \chi_{1}(.) \in \mathcal{X}\left(x_{0}, \downarrow_{\tau}^{t^{\prime}}\right), \exists \chi_{2}(.) \in \mathcal{X}\left(\chi\left(t^{\prime}\right), \uparrow_{t^{\prime}}^{\tau}\right)$ such that $\chi(t)=\left(\chi_{1} \cdot \chi_{2}\right)(t), \forall t \in \tau$;

$-\forall x_{0} \in X, \forall \tau_{1}, \tau_{2} \in[T]$, such that $\tau_{1} \cup \tau_{2} \in[T]$ and $\tau_{1} \cap \tau_{2}=\left\{t^{\prime}\right\}, \forall \chi_{1}(.) \in \mathcal{X}\left(x_{0}, \tau_{1}\right), \forall \chi_{2}(.) \in$ $\mathcal{X}\left(\chi_{1}\left(t^{\prime}\right), \tau_{2}\right), \exists \chi(.) \in \mathcal{X}\left(x_{0}, \tau_{1} \cup \tau_{2}\right)$ such that $\chi(t)=\left(\chi_{1} \cdot \chi_{2}\right)(t), \forall t \in \tau_{1} \cup \tau_{2} ;$

- $w: X \rightarrow W$ is a function that represents the interaction of the system with all the rest of the environment.

Remark 1: Abstract state systems are general enough to include as special cases, linear and non-linear dynamical control systems [15], [16], Kripke structures [2], finite state machines [1], hybrid systems [17], as well as infinitedimensional systems. For example, the following linear system in pencil form,

$$
\Sigma:\left\{\begin{array}{l}
E \dot{x}=A x, x \in X, \\
w=H x, w \in W,
\end{array}\right.
$$

can be represented by an abstract state system $\Sigma^{\prime}=$
$\left(X, W, \mathcal{X}^{\prime}, w\right)$, where for any initial condition $x_{0} \in X$ and for any time path $\tau \in\left[\mathbb{R}_{0}^{+}\right], \mathcal{X}^{\prime}\left(x_{0}, \tau\right)=\{\chi: \tau \rightarrow X \mid$ $\left.\chi \in \mathcal{C}^{1}(X), \chi\left(t_{0}^{\tau}\right)=x_{0}, E \dot{\chi}(t)=A \chi(t), \forall t \in \tau\right\}$.

In the following of the paper it will be of interest also to consider some special classes of abstract state systems. An abstract state system $\Sigma=(X, W, \mathcal{X}, w)$ is said to be: finite, if the cardinality of $X$ is finite; deterministic if, for any $x_{0} \in X$, any $\tau \in[T]$ and any $\chi_{1}(),. \chi_{2}(.) \in \mathcal{X}\left(x_{0}, \tau\right)$, $w\left(\chi_{1}(t)\right)=w\left(\chi_{2}(t)\right), \forall t \in \tau$ implies $\chi_{1}(t)=\chi_{2}(t), \forall t \in \tau$. Moreover a deterministic abstract state system is said to be autonomous if for any given $x_{0} \in X$ and any $\tau \in[T]$, the cardinality of $\mathcal{X}\left(x_{0}, \tau\right)$ is a most one.

Given an abstract state system $\Sigma=(X, W, \mathcal{X}, w)$, sometimes it is useful to split the manifest variables $w \in W$ into two or more components, such that $w=\left(w_{1}, w_{2}, \ldots\right)$ and $W=W_{1} \times W_{2} \times \ldots$ to distinguish the different meanings of manifest variables [22], [19], as for example variables that are accessible for control action etc.

We can now introduce the notion of interconnection between abstract state systems. The following definition is inspired by the notion of interconnection of Discrete Event Systems (DESs), that is a well-established issue in the theory of Supervisory Control (e.g. [13], [14]) and is based on the synchronization of the events driving the transitions in the interconnected DESs.

Definition 2: Given two abstract state systems $\Sigma_{1}=\left(X_{1}\right.$, $\left.W_{1} \times Z, \mathcal{X}_{1},\left(w_{1}, z_{1}\right)\right), \Sigma_{2}=\left(X_{2}, Z, \mathcal{X}_{2}, z_{2}\right)$, the interconnection $\Sigma_{1} \|_{Z} \Sigma_{2}$ between $\Sigma_{1}$ and $\Sigma_{2}$ in the shared variables set $Z$, is an abstract state system $(X, W, \mathcal{X}, w)$ where: $X=$ $X_{1} \times X_{2} ; W=W_{1} ; \mathcal{X}\left(\left(x_{10}, x_{20}\right), \tau\right)=\left\{\left(\chi_{1}(),. \chi_{2}().\right) \in\right.$ $\left.\mathcal{X}_{1}\left(x_{10}, \tau\right) \times \mathcal{X}_{2}\left(x_{20}, \tau\right) \mid z_{1}\left(\chi_{1}(t)\right)=z_{2}\left(\chi_{2}(t)\right), \forall t \in \tau\right\}$, $\forall\left(x_{10}, x_{20}\right) \in X, \forall \tau \in[T] ; w\left(\left(x_{1}, x_{2}\right)\right)=w_{1}\left(x_{1}\right)$, $\forall\left(x_{1}, x_{2}\right) \in X$.

Given any set $\Omega \subset \Omega_{1} \times \Omega_{2}$, denote by $\left.\Pi\right|_{\Omega_{i}}(\Omega)$, $i=1,2$ the projection of $\Omega$ onto $\Omega_{i}$, i.e. $\left.\Pi\right|_{\Omega_{i}}(\Omega)=$ $\left\{\omega_{i} \in \Omega_{i} \mid\left(\omega_{1}, \omega_{2}\right) \in \Omega\right\}, i=1,2$. Given two abstract state systems $\Sigma_{1}$ and $\Sigma_{2}$, interconnected as in Definition 2, let us define the following tuples: $\left.\Pi\right|_{\Sigma_{1}}\left(\Sigma_{1}||_{Z} \Sigma_{2}\right):=\left(\left.\Pi\right|_{X_{1}}(X)\right.$, $\left.W_{1} \times Z,\left.\quad \Pi\right|_{\mathcal{X}_{1}}(\mathcal{X}), \quad\left(w_{1}, z_{1}\right)\right),\left.\quad \Pi\right|_{\Sigma_{2}}\left(\left.\Sigma_{1}\right|_{W} \Sigma_{2}\right) \quad:=$ $\left(\left.\Pi\right|_{X_{2}}(X), Z,\left.\Pi\right|_{\mathcal{X}_{2}}(\mathcal{X}), z_{2}\right)$, that represent the 'projection' of $\Sigma_{1} \|_{Z} \Sigma_{2}$ onto $\Sigma_{1}$ and $\Sigma_{2}$ respectively.

Proposition 1: The tuples $\left.\Pi\right|_{\Sigma_{1}}\left(\Sigma_{1} \|_{Z} \Sigma_{2}\right)$ and $\left.\Pi\right|_{\Sigma_{2}}\left(\Sigma_{1} \|_{W} \Sigma_{2}\right)$ are abstract state systems.

Moreover, it is easy to see that,

Proposition 2: $\Sigma_{1}\left\|_{Z}\left(\left.\Pi\right|_{\Sigma_{2}}\left(\Sigma_{1} \|_{Z} \Sigma_{2}\right)\right)=\Sigma_{1}\right\|_{Z} \Sigma_{2}$.

\section{B. The achievable bisimilar behaviour problem}

This section is devoted to the problem setting of this paper. We start with formally defining the notion of bisimulation of abstract state systems.

Definition 3: Given two abstract state systems $\Sigma_{i}=$ $\left(X_{i}, W_{i} \times Z, \mathcal{X}_{i},\left(w_{i}, z_{i}\right)\right), i=1,2$, a set $\mathcal{S} \subset X_{1} \times X_{2}$, is a simulation relation of $\Sigma_{2}$ by $\Sigma_{1}$ w.r.t. $Z$ if for any $\left(x_{01}, x_{02}\right) \in \mathcal{S}$, for any time path $\tau \in[T]$, for any solution $\chi_{2}(.) \in \mathcal{X}_{2}\left(x_{20}, \tau\right)$, there exists a solution $\chi_{1}(.) \in$ $\mathcal{X}_{1}\left(x_{10}, \tau\right)$ such that: $\left(\chi_{1}(t), \chi_{2}(t)\right) \in \mathcal{S}, z_{1}\left(\chi_{1}(t)\right)=$ $z_{2}\left(\chi_{2}(t)\right), \forall t \in \tau$. Moreover $\Sigma_{2}$ is simulated by $\Sigma_{1}$ or 
equivalently $\Sigma_{1}$ simulates $\Sigma_{2}$ w.r.t. $Z$, denoted $\Sigma_{2} \lesssim_{Z} \Sigma_{1}$, if there exists a simulation relation $\mathcal{S}$ w.r.t. $Z$, such that $\left.\Pi\right|_{X_{2}}(\mathcal{S})=X_{2}$.

Definition 4: Given two abstract state systems $\Sigma_{i}=$ $\left(X_{i}, W_{i} \times Z, \mathcal{X}_{i},\left(w_{i}, z_{i}\right)\right), i=1,2$, a set $\mathcal{B} \subset X_{1} \times X_{2}$, is a bisimulation relation between $\Sigma_{1}$ and $\Sigma_{2}$ w.r.t. $Z$ if $\mathcal{B}$ is a simulation relation of $\Sigma_{1}$ by $\Sigma_{2}$ and of $\Sigma_{2}$ by $\Sigma_{1}$, w.r.t. $Z$. Moreover $\Sigma_{1}$ is bisimilar to $\Sigma_{2}$ w.r.t. $Z$, denoted $\Sigma_{1} \approx_{Z}$ $\Sigma_{2}$, if there exists a bisimulation relation $\mathcal{B}$ w.r.t. $Z$ such that $\left.\Pi\right|_{X_{i}}(\mathcal{B})=X_{i}, i=1,2$.

Remark 2: Definitions 3 and 4 are inspired by the notions of simulation and bisimulation of concurrent processes, as introduced by Milner [7] and Park [9], and unify the notions of simulation and bisimulation given for Kripke formulas [2], linear and non-linear dynamical systems introduced in [21], and switching systems introduced in [10].

Remark 3: While

$$
\Sigma_{1} \approx_{W} \Sigma_{2} \Rightarrow\left(\Sigma_{1} \lesssim W \Sigma_{2} \text { and } \Sigma_{2} \lesssim W \Sigma_{1}\right),
$$

the converse implication does not hold for the general class of abstract state systems (see some classical counterexamples for Kripke formulas in [2].) On the other hand, the converse implication of (2) is proved in [21] to be true for the special class of finite-dimensional non-deterministic linear systems.

Remark 4: The notion of bisimilarity is an equivalence relation on the space of abstract state systems, since it satisfies the reflexivity, symmetry and transitivity properties. On the other hand, the notion of simulation is not an equivalence notion on the space of abstract state systems, since the symmetry property is not satisfied, while reflexivity and transitivity are fulfilled.

Remark 5: One of the most popular application of the bisimulation theory is the reduction, in terms of 'size', of the system under consideration. The basic idea is to find a bisimulation relation $\mathcal{B}$ between an abstract state system $\Sigma$ and itself and then to factorize the state space $X$ of $\Sigma$ by the equivalence relation, on $X$ induced by $\mathcal{B}$.

We expect that an algebraic characterization of the notions of bisimulation and simulation depend strongly on the models under consideration. For example, a complete algebraic characterization of non-deterministic linear and non-linear dynamical systems has been developed in [21], and for switching systems in [10]. Given two abstract state systems $\Sigma_{i}=\left(X_{i}, W_{i} \times Z, \mathcal{X}_{i},\left(w_{i}, z_{i}\right)\right), i=1,2$, the maximal simulation relation of $\Sigma_{1}$ by $\Sigma_{2}$ w.r.t. $Z$, is that simulation relation $\mathcal{S}_{Z}^{*}\left(\Sigma_{1}, \Sigma_{2}\right)$ such that for any simulation relation $\mathcal{S}$ of $\Sigma_{1}$ by $\Sigma_{2}$ w.r.t. $Z, \mathcal{S} \subset \mathcal{S}_{Z}^{*}\left(\Sigma_{1}, \Sigma_{2}\right)$; moreover the maximal bisimulation relation between $\Sigma_{1}$ and $\Sigma_{2}$ w.r.t. $Z$, is that bisimulation relation $\mathcal{B}_{Z}^{*}\left(\Sigma_{1}, \Sigma_{2}\right)$ such that for any bisimulation relation $\mathcal{B}$ between $\Sigma_{1}$ and $\Sigma_{2}$ w.r.t. $Z$, $\mathcal{B} \subset \mathcal{B}_{Z}^{*}\left(\Sigma_{1}, \Sigma_{2}\right)$.

Theorem 1: Given a pair of abstract state systems $\Sigma_{i}=$ $\left(X_{i}, W_{i} \times Z, \mathcal{X}_{i},\left(w_{i}, z_{i}\right)\right), i=1,2, \mathcal{S}_{Z}^{*}\left(\Sigma_{1}, \Sigma_{2}\right)$ and $\mathcal{B}_{Z}^{*}\left(\Sigma_{1}, \Sigma_{2}\right)$ exist.

Remark 6: The computation of the maximal bimulation and simulation relations is expected to depend strongly on the semantics of the models under consideration. For example, algorithms converging in a finite number of steps to the maximal bisimulation relation between finite Kripke formulas can be found in [2], between non-deterministic linear and non-linear dynamical control systems in [21] and between switching systems in [10].

We can now formalize the problem that we focus on in this paper:

Problem 1: (Achievable bisimilar behaviour problem) Given a plant abstract state system $\mathcal{P}=\left(X_{p}, W \times Z\right.$, $\left.\mathcal{X}_{p},\left(w_{p}, z_{p}\right)\right)$ and a desired abstract state system $\mathcal{Q}=$ $\left(X_{q}, W, \mathcal{X}_{q}, w_{q}\right)$, find conditions for which there exists a controller abstract state system $\mathcal{C}=\left(X_{c}, Z, \mathcal{X}_{c}, z_{c}\right)$, such that $\mathcal{P} \|_{Z} \mathcal{C} \approx_{W} \mathcal{Q}$.

The achievable bisimilar behaviour problem is in fact very close to what addressed in [19] that studies conditions for which, a plant system $\mathcal{P}$ interconnected with a controller $\mathcal{C}$, was external behaviour equivalent to a desired system $\mathcal{Q}$. The notion of external behaviour equivalence is very close to the notion of bisimilarity for deterministic systems and at least, for the classes of deterministic finite-dimensional linear systems and of deterministic transition systems, the two equivalence notions are proved in [21] and respectively in [2] to coincide: therefore for those classes of deterministic systems, results of [19] solve in fact also the achievable bisimilar behaviour problem. On the other hand, when dealing with non-deterministic abstract state systems, while bisimilar equivalence implies external behaviour equivalence, there are several examples in the literature showing that the converse is not true in general (see [21] for the class of linear dynamical systems and [2] for the class of Kripke structures) and this motivates the results of this paper.

\section{BISIMILAR AND SIMILAR INTERCONNECTED ABSTRACT STATE SYSTEMS}

Aim of this section is to characterize what are the relationships of bisimulation/simulation equivalences of interconnected abstract state systems, on the basis of the bisimulation/simulation equivalence properties of the preconnected abstract state systems.

\section{A. Interconnection preserves bisimulation and simulation equivalences}

In this section, we prove that interconnection does preserve bisimulation and simulation equivalences, or in other words that the so-called congruence property is fulfilled.

Theorem 2: Consider four abstract state systems $\Sigma_{1}=$ $\left(X_{1}, Z, \mathcal{X}_{1}, z_{1}\right), \Sigma_{2}=\left(X_{2}, Z, \mathcal{X}_{2}, z_{2}\right), \Sigma_{3}=\left(X_{3}, W \times\right.$ $\left.Z, \mathcal{X}_{3},\left(w_{3}, z_{3}\right)\right), \Sigma_{4}=\left(X_{4}, W \times Z, \mathcal{X}_{4},\left(w_{4}, z_{4}\right)\right)$. If $\Sigma_{2} \lesssim_{Z}$ $\Sigma_{1}$ and $\Sigma_{4} \lesssim_{W \times Z} \Sigma_{3}$, then $\left(\Sigma_{4} \|_{Z} \Sigma_{2}\right) \lesssim_{W}\left(\Sigma_{3} \|_{Z} \Sigma_{1}\right)$. If $\Sigma_{1} \approx_{Z} \quad \Sigma_{2}$ and $\Sigma_{3} \approx_{W \times Z} \quad \Sigma_{4}$, then $\left(\Sigma_{3} \|_{Z} \Sigma_{1}\right) \approx_{W}$ $\left(\Sigma_{4} \|_{Z} \Sigma_{2}\right)$.

Remark 7: The result above is important to a compositional modeling and control of abstract state systems as argued in [18] for the class of hybrid systems. Suppose for instance, that the purpose is to design a controller $\Sigma_{1}$ to be applied to an abstract state system $\Sigma_{2}$, for ensuring some prescribed performances. Then, one can consider an abstract 
state system $\Sigma_{2}^{r e d}$, obtained by reducing $\Sigma_{2}$ by bisimulation (cf. Remark 5), and then to design a controller $\Sigma_{1}$ for the 'smaller' abstract state system $\Sigma_{2}^{r e d}$ : Theorem 2 ensures that $\Sigma_{2}^{r e d} \|_{Z} \Sigma_{1}$ is bisimilar to $\Sigma_{2} \|_{Z} \Sigma_{1}$.

\section{B. Elimination of redundant variables in interconnection}

When interconnecting a pair of systems, the resulting system is characterized by some redundant internal state variables that takes into account the interconnection between the systems under consideration. A well-known result in the context of linear differential systems is the so-called Elimination Theorem [12], that allows a 'reduction' of the redundant variables. The following result can be seen as an extension of the Elimination Theorem to the context of abstract state systems and of the notion of bisimulation.

Proposition 3: Given two abstract state systems $\Sigma_{1}=$ $\left(X_{1}, W \times Z, \mathcal{X}_{1},\left(w_{1}, z_{1}\right)\right)$ and $\Sigma_{2}=\left(X_{2}, Z, \mathcal{X}_{2}, z_{2}\right)$, then $\left.\Pi\right|_{\Sigma_{1}}\left(\Sigma_{1} \|_{Z} \Sigma_{2}\right) \approx_{W}\left(\Sigma_{1} \|_{Z} \Sigma_{2}\right)$.

Moreover as a consequence,

Corollary 1: Consider four abstract state systems $\Sigma_{1}=$ $\left(X_{1}, W \times Z, \mathcal{X}_{1},\left(w_{1}, z_{1}\right)\right), \Sigma_{2}=\left(X_{2}, W \times Z, \mathcal{X}_{2},\left(w_{2}, z_{2}\right)\right)$, $\Sigma_{3}=\left(X_{3}, Z, \mathcal{X}_{3}, z_{3}\right), \quad \Sigma_{4}=\left(X_{4}, Z, \mathcal{X}_{4}, z_{4}\right)$ such that $\Sigma_{1}\left\|_{Z} \Sigma_{3} \quad \approx_{W} \quad \Sigma_{2}\right\|_{Z} \Sigma_{4}$. Then $\left.\Pi\right|_{\Sigma_{1}}\left(\Sigma_{1} \|_{Z} \Sigma_{3}\right) \approx_{W}$ $\left.\Pi\right|_{\Sigma_{2}}\left(\Sigma_{2}|| \Sigma^{\Sigma_{4}}\right)$.

\section{Bisimulation equivalence and interconnection}

There is a deep connection between the notions of bisimulation and of interconnection of abstract state systems. The next results highlight those connections.

Proposition 4: Given two abstract state systems $\Sigma_{1}=$ $\left(X_{1}, W_{1} \times Z, \mathcal{X}_{1},\left(w_{1}, z_{1}\right)\right)$ and $\Sigma_{2}=\left(X_{2}, Z, \mathcal{X}_{2}, z_{2}\right)$. Then, $\left.\left.\Pi\right|_{\Sigma_{1}}(\Sigma) \approx_{Z} \Pi\right|_{\Sigma_{2}}(\Sigma)$ and $X=\mathcal{B}_{Z}^{*}\left(\left.\Pi\right|_{\Sigma_{1}}(\Sigma),\left.\Pi\right|_{\Sigma_{2}}(\Sigma)\right)$, where $\Sigma=\Sigma_{1} \|_{Z} \Sigma_{2}:=(X, W, \mathcal{X}, w)$.

Moreover,

Proposition 5: Two abstract state systems $\Sigma_{1}=\left(X_{1}\right.$, $\left.W_{1} \times Z, \mathcal{X}_{1},\left(w_{1}, z_{1}\right)\right)$ and $\Sigma_{2}=\left(X_{2}, Z, \mathcal{X}_{2}, z_{2}\right)$ are bisimilar w.r.t. $Z$ if and only if $\left.\Pi\right|_{\Sigma_{1}}\left(\Sigma_{1} \|_{Z} \Sigma_{2}\right)=\Sigma_{1}$ and $\left.\Pi\right|_{\Sigma_{2}}\left(\Sigma_{1}||_{Z} \Sigma_{2}\right)=\Sigma_{2}$.

We conclude this section, by giving the following result.

Proposition 6: Consider three abstract state systems $\Sigma_{1}=$ $\left(X_{1}, W \times Z, \mathcal{X}_{1},\left(w_{1}, z_{1}\right)\right), \Sigma_{2}=\left(X_{2}, Z, \mathcal{X}_{2}, z_{2}\right), \Sigma_{3}=$ $\left(X_{3}, W, \mathcal{X}_{3}, w_{3}\right)$ such that $\Sigma_{1} \|_{Z} \Sigma_{2} \approx_{W} \Sigma_{3}$. Then, $\left(\left.\Pi\right|_{\Sigma_{1}}\right.$ $\left.\left(\Sigma_{1} \|_{Z} \Sigma_{2}\right)\right) \|\left._{W} \Sigma_{3} \approx_{Z} \Pi\right|_{\Sigma_{2}}\left(\Sigma_{1} \|_{Z} \Sigma_{2}\right)$.

As a consequence,

Corollary 2: Consider three abstract state systems $\Sigma_{1}=$ $\left(X_{1}, W \times Z, \mathcal{X}_{1},\left(w_{1}, z_{1}\right)\right), \Sigma_{2}=\left(X_{2}, Z, \mathcal{X}_{2}, z_{2}\right), \Sigma_{3}=$ $\left(X_{3}, W, \mathcal{X}_{3}, w_{3}\right)$ such that $\Sigma_{1} \|_{Z} \Sigma_{2} \quad \approx_{W} \quad \Sigma_{3}$. Then $\left.\Pi\right|_{\Sigma_{2}}\left(\Sigma_{1} \|_{Z} \Sigma_{2}\right) \lesssim_{Z}\left(\Sigma_{1} \|_{W} \Sigma_{3}\right)$.

\section{GEnERAL RESUlTS}

In this section we give some sufficient and some necessary conditions for solving the achievable bisimilar behaviour problem for the general class of abstract state systems.

A basic sufficient condition for assessing the solvability of the achievable bisimilar behaviour problem is that:

$$
\mathcal{P} \|_{Z}\left(\mathcal{P} \|_{W} \mathcal{Q}\right) \approx_{W} \mathcal{Q}
$$

Condition above makes use of the controller $\mathcal{C}_{\text {can }}=$ $\left(X_{\text {can }}, Z, \mathcal{X}_{\text {can }}, z_{\text {can }}\right):=\mathcal{P} \|_{W} \mathcal{Q}$, that is known in the literature (e.g. [8], [19], [5]) as the canonical controller. The canonical controller was introduced in the context of network interconnection structures appearing in [8], and after that a research was carried out by the control system community along this research direction (e.g. [19], [5].) Condition (3) will be shown in Theorem 7 to be also necessary for solving the achievable bisimilar behaviour of non-deterministic linear dynamical systems. Moreover,

Theorem 3: The achievable bisimilar behaviour problem is solvable only if

On the other hand,

$$
\mathcal{Q} \lesssim_{W} \mathcal{P} \|_{Z} \mathcal{C}_{\text {can }}
$$

Remark 8: There are some counterexamples in the literature showing that the converse implication of (4),

$$
\mathcal{P} \|_{Z} \mathcal{C}_{\text {can }} \lesssim W \mathcal{Q}
$$

is not necessary for solving the achievable bisimilar behaviour problem in the case of deterministic systems (e.g. [19]) and therefore in the more general case of nondeteministic abstract state systems. Note that even if condition (5) were satisfied, then the combination of conditions (4) and (5) does not imply $\mathcal{P} \|_{Z} \mathcal{C}_{c a n} \approx_{W} \mathcal{Q}$, as pointed out in Remark 3, for the general case of abstract state systems.

Theorem 3 and Remark 8 suggest that the canonical controller $\mathcal{C}_{\text {can }}$ is in some way 'too big' for solving the achievable bisimilar behaviour problem and therefore in the following we look for a weaker condition than that one in (3). More precisely inspired by [5], we will define a controller $\mathcal{C}_{\text {can }}^{*}$, that is a candidate to solve the achievable bisimilar behaviour problem and that is 'smaller' than $\mathcal{C}_{\text {can }}$. Given an abstract state system $\Sigma=(X, W, \mathcal{X}, w)$ and a set $\bar{X} \subset X$ define the restriction of $\Sigma$ to $\bar{X}$ as that abstract state system $\left.\Sigma\right|_{\bar{X}}$ whose state state is $\bar{X}$ and whose dynamics coincide with that one of $\Sigma$ that makes $\bar{X}$ invariant, i.e. $\left.\Sigma\right|_{\bar{X}}=(\bar{X}, W, \overline{\mathcal{X}}, \bar{w})$ where: $\overline{\mathcal{X}}\left(x_{0}, \tau\right)=\left\{\chi(.) \in \mathcal{X}\left(x_{0}, \tau\right) \mid\right.$ $\chi(t) \in \bar{X}, \forall t \in \tau\}, \forall x_{0} \in \bar{X}, \forall \tau \in[T] ; \bar{w}: \bar{X} \rightarrow W$ such that $\bar{w}\left(x_{0}\right)=w\left(x_{0}\right), \forall x_{0} \in \bar{X}$.

The following two results give some properties of the restriction of abstract state systems, as defined above.

Proposition 7: Given an abstract state system $\Sigma=$ $(X, W, \mathcal{X}, w)$ and a set $\bar{X} \subset X,\left.\Sigma\right|_{\bar{X}} \lesssim_{W} \Sigma$.

Proposition 8: Given two abstract state systems $\Sigma_{i}=$ $\left(X_{i}, W, \mathcal{X}_{i}, w_{i}\right), i=1,2,\left.\left.\Sigma_{1}\right|_{\bar{X}_{1}} \approx_{W} \Sigma_{2}\right|_{\bar{X}_{2}}$, where $\bar{X}_{i}=$ $\left.\Pi\right|_{X_{i}}\left(\mathcal{B}_{W}^{*}\left(\Sigma_{1}, \Sigma_{2}\right)\right), i=1,2$.

We can now propose the controller $\mathcal{C}_{\text {can }}^{*}$. Let $\Lambda: 2^{X_{\text {can }}} \rightarrow$ $2^{X_{p}}$ be such that for any given $X_{c} \subset X_{c a n}, \Lambda\left(X_{c}\right):=$ $\bigcup_{x_{c} \in X_{c}} \Lambda\left(\left\{x_{c}\right\}\right)$ and for any given $x_{c} \in X_{c}$,

$$
\Lambda\left(\left\{x_{c}\right\}\right):=\left\{\begin{array}{c}
x_{p} \in X_{p} \mid \forall \tau \in[T], \\
\exists \chi_{c}(.) \in \mathcal{X}_{c a n}\left(x_{c}, \tau\right), \\
\exists \chi_{p}(.) \in \mathcal{X}_{p}\left(x_{p}, \tau\right): \\
z_{c a n}\left(\chi_{c}(t)\right)=z_{p}\left(\chi_{p}(t)\right), \\
\chi_{c}(t) \in X_{c}, \forall t \in \tau
\end{array}\right\} .
$$

The map $\Lambda\left(X_{c}\right)$ captures all the states of the plant $\mathcal{P}$, that are interconnectable with a state of the controller $\left.\mathcal{C}_{c a n}\right|_{X_{c}}$ 
and in fact $\left(x_{p}, x_{c}\right)$ is a state of $\mathcal{P} \|_{Z}\left(\left.\mathcal{C}_{c a n}\right|_{X_{c}}\right)$, if and only if $x_{p} \in \Lambda\left(\left\{x_{c}\right\}\right)$. Set

$$
X_{p}^{*}=\left.\Pi\right|_{X_{p}}\left(\mathcal{B}_{W}^{*}\left(\mathcal{Q}, \mathcal{P} \|_{Z} \mathcal{C}_{c a n}\right)\right),
$$

and let $X_{c}^{*}$ be the maximal subset $X_{c}$ of $X_{c a n}$ such that $\Lambda\left(X_{c}\right) \subset X_{p}^{*}$; finally set $\mathcal{C}_{c a n}^{*}:=\left.\mathcal{C}_{\text {can }}\right|_{X_{c}^{*}}$.

Proposition 9: The achievable bisimilar behaviour problem is solvable if:

$$
\mathcal{P} \|_{Z} \mathcal{C}_{c a n}^{*} \approx{ }_{W} \mathcal{Q}
$$

Condition (7) will be shown in Theorem 5 to be also necessary for solving the achievable bisimilar behaviour problem in the case of autonomous abstract state systems.

We conclude this section by highlighting some properties of the class of controllers ensuring a solution to the achievable bisimilar behaviour problem.

Proposition 10: If a controller $\mathcal{C}$ solves the achievable bisimilar behaviour problem, then

$$
\left.\left.\Pi\right|_{\mathcal{C}}\left(\left.\mathcal{P}\right|_{Z} \mathcal{C}\right) \approx_{Z} \mathcal{C}_{c a n}\right|_{X_{c}}
$$

where $\left.X_{c}=\mathcal{B}_{W}^{*}\left(\left.\Pi\right|_{\mathcal{P}}\left(\mathcal{P} \|_{Z} \mathcal{C}\right), \mathcal{Q}\right)\right)$. Moreover $\left.\mathcal{C}_{c a n}\right|_{X_{c}}$ solves the achievable bisimilar behaviour problem.

A direct consequence of the result above is that,

Theorem 4: The achievable bisimilar behaviour problem is solvable if and only if there exists $X_{c} \subset X_{c a n}$ such that $\left(\mathcal{P} \|_{Z}\left(\left.\mathcal{C}_{c a n}\right|_{X_{c}}\right)\right) \approx_{W} \mathcal{Q}$.

By Proposition 10, one can uniquely associate to any solution $\mathcal{C}$ of the achievable bisimilar behaviour problem a controller $\left.\mathcal{C}_{c a n}\right|_{X_{c}}$, for some subset $X_{c}$ of $X_{c a n}$, that we call canonical representative of $\mathcal{C}$. The space of canonical representatives is a poset with respect to the partial ordering $\prec$ defined by $\left.\left.\mathcal{C}_{c a n}\right|_{X_{c}^{1}} \prec \mathcal{C}_{c a n}\right|_{X_{c}^{2}}$, if $X_{c}^{1} \subset X_{c}^{2} \subset X_{c a n}$ and by Proposition 10, is 'upper bounded' by $\mathcal{C}_{c a n}$, i.e. for any canonical representative $\left.\mathcal{C}_{c a n}\right|_{X_{c}},\left.\mathcal{C}_{c a n}\right|_{X_{c}} \prec \mathcal{C}_{c a n}$.

\section{Special Classes of abstract state systems}

This section is devoted to the specialization of the theory developed for general abstract state systems to some important sub-classes of abstract state systems.

\section{A. Autonomous abstract state systems}

If the plant $\mathcal{P}$ and the desired system $\mathcal{Q}$ are autonomous, then the canonical controller $\mathcal{C}_{c a n}$ is also autonomous. The following result stresses a property of the maximal bisimulation relations between $\mathcal{P} \|{ }_{Z}\left(\left.\mathcal{C}_{c a n}\right|_{X_{c}^{1}}\right)$ and $\mathcal{Q}$ and between $\left.\mathcal{P}\right|_{Z}\left(\left.\mathcal{C}_{c a n}\right|_{X_{c}^{2}}\right)$ and $\mathcal{Q}$, where $\left.\mathcal{C}_{c a n}\right|_{X_{c}^{1}}$ and $\left.\mathcal{C}_{c a n}\right|_{X_{c}^{2}}$ are canonical representatives.

Proposition 11: If $\mathcal{P}$ and $\mathcal{Q}$ are autonomous, then for any $X_{c}^{1} \subset X_{c}^{2} \subset X_{c a n}, \mathcal{B}_{W}^{*}\left(\left(\mathcal{P} \|_{Z}\left(\left.\mathcal{C}_{c a n}\right|_{X_{c}^{1}}\right)\right), \mathcal{Q}\right)=$ $\mathcal{B}_{W}^{*}\left(\left(\mathcal{P} \|_{Z}\left(\left.\mathcal{C}_{c a n}\right|_{X_{c}^{2}}\right)\right), \mathcal{Q}\right) \cap\left(X_{p, c}^{1} \times X_{q}\right)$, where $X_{p, c}^{1}$ is the state space of $\mathcal{P}\|\|_{Z}\left(\left.\mathcal{C}_{c a n}\right|_{X_{c}^{1}}\right)$.

A direct consequence of the result above is that the map associating to any $X_{c} \subset X_{c a n}$ the set $\mathcal{B}_{W}^{*}\left(\mathcal{P} \|_{Z}\left(\left.\mathcal{C}_{c a n}\right|_{X_{c}}\right), \mathcal{Q}\right)$ is 'monotone' with respect to set inclusion.

Corollary 3: If $\mathcal{P}$ and $\mathcal{Q}$ are autonomous then, for any $X_{c}^{1} \subset X_{c}^{2} \subset X_{c a n}, \mathcal{B}_{W}^{*}\left(\left(\mathcal{P} \|_{Z}\left(\left.\mathcal{C}_{c a n}\right|_{X_{c}^{1}}\right)\right), \mathcal{Q}\right) \subset$ $\mathcal{B}_{W}^{*}\left(\left(\mathcal{P}||_{Z}\left(\left.\mathcal{C}_{c a n}\right|_{X_{c}^{2}}\right)\right), \mathcal{Q}\right)$.
Moreover the following result shows that, for the special class of autonomous abstract state systems, the space of canonical representatives is upper bounded by $\mathcal{C}_{\text {can }}^{*}$.

Proposition 12: If $\mathcal{P}$ and $\mathcal{Q}$ are autonomous, then for any canonical representative $\left.\mathcal{C}_{c a n}\right|_{X_{c}}, X_{c} \subset X_{c}^{*}$.

We can now give the main result of this section.

Theorem 5: The achievable bisimilar behaviour problem, where the abstract state systems $\mathcal{P}$ and $\mathcal{Q}$ are autonomous, is solvable if and only if $\mathcal{P} \|_{Z} \mathcal{C}_{c a n}^{*} \approx_{W} \mathcal{Q}$.

\section{B. Finite abstract state systems}

If the plant $\mathcal{P}$ and the desired system $\mathcal{Q}$ are finite then, the canonical controller $\mathcal{C}_{c a n}$ is also finite. Moreover, by applying Theorem 4 to this special case, a procedure can be developed for finding a solution to the achievable bisimilar behaviour problem, by checking for any $X_{c} \subset X_{c a n}$, if the controller $\left.\mathcal{C}_{c a n}\right|_{X_{c}}$ is a solution to the achievable bisimilar behaviour problem. This approach is very important from the decidability point of view, as shown in the following.

Theorem 6: The achievable bisimilar behaviour problem, for the class of finite abstract state systems, is decidable in at most a finite number of steps,

$$
N^{*} \leq \sum_{k=1}^{N}\left(\begin{array}{c}
N \\
k
\end{array}\right),
$$

where $\left(\begin{array}{c}N \\ i\end{array}\right)$ is the Newton binomial coefficient and $N$ is the cardinality of $X_{c a n}$.

\section{Non-deterministic linear systems}

We focus on here the class of linear systems, whose dynamics are given by means of differential-algebraic equations [6], i.e. systems $\Sigma$ of the form (1), where $X$ and $W$ are finite-dimensional linear spaces and $E, A$ and $H$ are matrices of appropriate dimensions. A linear system of the form (1) can be seen as a particular abstract state system $\Sigma=(X, W, \mathcal{X}, w)$, as pointed out in Remark 1 .

Remark 9: Linear systems of the form (1) are in general non-deterministic and generalize the usual notion of nondeterministic finite-dimensional linear system [15].

In the following we focus on the achievable bisimilar behaviour problem where, the systems under consideration are as in (1). More precisely,

Problem 2: (Linear achievable bisimilar behaviour problem) Given a plant system,

$$
\mathcal{P}:\left\{\begin{array}{l}
E_{p} \dot{x}_{p}=A_{p} x_{p}, x_{p} \in X_{p}, \\
w_{p}=H_{p} x_{p}, w_{p} \in W, \\
z_{p}=K_{p} x_{p}, z_{p} \in Z,
\end{array}\right.
$$

and a desired system

$$
\mathcal{Q}:\left\{\begin{array}{l}
E_{q} \dot{x}_{q}=A_{q} x_{q}, x_{q} \in X_{q}, \\
w_{q}=H_{q} x_{q}, w_{q} \in W,
\end{array}\right.
$$

find conditions for which there exists a controller

$$
\mathcal{C}:\left\{\begin{array}{l}
E_{c} \dot{x}_{c}=A_{c} x_{c}, x_{c} \in X_{c} \\
z_{c}=K_{c} x_{c}, z_{c} \in Z
\end{array}\right.
$$


such that the interconnected system

$$
\mathcal{P} \|_{Z} \mathcal{C}:\left\{\begin{array}{l}
E_{p} \dot{x}_{p}=A_{p} x_{p}, x_{p} \in X_{p}, \\
E_{c} \dot{x}_{c}=A_{c} x_{c}, x_{c} \in X_{c}, \\
K_{p} x_{p}=K_{c} x_{c}, \\
w_{p}=H_{p} x_{p},
\end{array}\right.
$$

is bisimilar to $\mathcal{Q}$ w.r.t. $W$.

In view of Remark 1, the results of Section IV hold for the class of systems that we are considering in this section. Moreover, given the particular structure of linear systems, a stronger result can be stated. In the following we will show that the sufficient condition (3) is also necessary for solving the linear achievable bisimilar behaviour problem. Given a plant $\mathcal{P}=\left(X_{p}, W, \mathcal{X}_{p}, w_{p}\right)$ of the form (1), let us define the state-constrained dynamical system $\mathcal{P}_{0}=\left(X_{p}^{0}, W, \mathcal{X}_{p}^{0}, w_{p_{0}}\right)$, characterized by the following dynamics:

$$
\mathcal{P}_{0}:\left\{\begin{array}{l}
E_{p} \dot{x}_{p_{0}}=A_{p} x_{p_{0}}, x_{p_{0}} \in X_{p}^{0}, \\
w_{p_{0}}=H_{p} x_{p_{0}},
\end{array}\right.
$$

where $X_{p}^{0}$ denotes the maximal invariant set contained in $\operatorname{ker}\left(H_{p}\right)$, i.e. $X_{p}^{0}:=\left\{x_{p_{0}} \in X_{p} \mid H_{p} \chi(t)=0, \forall t \in \tau, \forall \tau \in\right.$ $\left.\left[\mathbb{R}_{0}^{+}\right], \forall \chi(.) \in \mathcal{X}_{p}^{0}\left(x_{p_{0}}, \tau\right)\right\}$. The linear system $\mathcal{P}_{0}$ models all the hidden dynamics associated with $\mathcal{P}$. In order to solve the linear achievable bisimilar behaviour problem, one has to take into account the dynamics of $\mathcal{P}_{0}$, since they are internal modes of the plant $\mathcal{P}$ and therefore, they cannot be controlled by any controller $\mathcal{C}$; for this reason it is necessary to assume that the behaviour of $\mathcal{P}_{0}$, matches the specification given by the desired system $\mathcal{Q}$.

Proposition 13: Given a plant $\mathcal{P}$ and a desired system $\mathcal{Q}$, if $\mathcal{P} \|_{Z} \mathcal{C} \approx_{W} \mathcal{Q}$ for some controller $\mathcal{C}$, then $\mathcal{P}_{0} \lesssim{ }_{W} \mathcal{Q}$.

Moreover the following result shows that, if $\mathcal{Q}$ simulates the hidden dynamics of $\mathcal{P}$ (represented by $\mathcal{P}_{0}$ ), then $\mathcal{Q}$ simulates the overall system $\mathcal{P} \|_{Z}\left(\mathcal{P} \|_{W} \mathcal{Q}\right)$.

Lemma 1: If $\mathcal{P}_{0} \lesssim_{W} \mathcal{Q}$ then $\left(\mathcal{P} \|_{Z}\left(\mathcal{P} \|_{W} \mathcal{Q}\right)\right) \lesssim_{W} \mathcal{Q}$.

By combining Proposition 13 and Lemma 1, the following result holds.

Theorem 7: The linear achievable bisimilar behaviour problem is solvable if and only if $\mathcal{P} \|_{Z} \mathcal{C}_{c a n} \approx_{W} \mathcal{Q}$.

Conditions of Theorem 7 are checkable in a finite number of steps, in view of the results of [20]. Moreover, even though we are focusing here on continuous-time systems, Theorem 7 also holds for discrete-time linear systems. Furthermore, the results of this section do not make use of the finitedimensionality of the state space and manifest space of the systems under consideration. Therefore, Theorem 7 also holds for infinite-dimensional linear systems.

\section{CONCLUSiON}

We considered the achievable bisimilar behaviour problem. Some sufficient and necessary conditions were developed for the general class of non-deterministic abstract state systems and stronger results for characterizing the achievable bisimilar behaviour problem for the special classes of autonomous abstract state systems, finite abstract state systems and non-deterministic linear systems were found.

\section{REFERENCES}

[1] Barrett, G., Lafortune S., Bisimulation, the supervisory control problem and strong model matching for finite state machines, Discrete Event Syst.: Theory Appl. vol. 8, no. 4, pp. 377-429, Dec. 1998.

[2] Clarke, E. M., Grumberg, O., Peled, D. A., Model Checking, The MIT Press, Cambridge, Massachusetts, 2002.

[3] Di Benedetto, M. D., Grizzle, J. W., Asymptotic Model Matching for Nonlinear Systems, IEEE Trans. on Automatic Control, Vol. 39, no. 8, August 1994, pp. 1538-1550.

[4] Di Benedetto, M. D., Sangiovanni-Vincentelli, A., Model Matching for Finite-States Machines, IEEE Trans. on Automatic Control, Vol. 46, no. 11, November 2001, pp. 1726-1737.

[5] Julius, A. A., Willems, J. C., Belur, M. N., Trentelman, H.L., The canonical controllers and regular interconnection, Systems \& Control Letters, 8(54), pp 787-797, August 2005.

[6] Kuijper, M., First-Order Representations of Linear Systems, Birkhauser, Boston, 1994

[7] Milner, R., Comunication and Concurrency, Prentice Hall International Series in Computer Science, 1989.

[8] Narayanan, H., Some applications of an Implicit Duality Theorem to connections of structures of special types including Dirac and reciprocal structures, Systems \& Control Letters, 45 (2002) pp. 8796.

[9] Park, D., Concurrency and automata on infinite sequences. In P. Deussen, Editor, Fifth GI Conference on Theoretical Computer Science, vol. 104 of Lecture Notes in Computer Science, Springer 1981.

[10] Pola, G., van der Schaft, A. J., Di Benedetto, M. D., Bisimulation Theory for Switching Linear Systems, Proc. of the 43st IEEE Conference on Decision and Control (CDC 04), Paradise Island, Bahamas, USA, 14th - 17th December 2004, pp. 1406-1411.

[11] Pola, G., van der Schaft, A. J., Di Benedetto, M. D., Achievable Bisimilar Behaviour of Abstract State Systems, Department of Electrical Engineering \& Computer Science, University of L'Aquila, Italy, Research Report, September 2005 http://www.diel.univaq.it/tr/web/web_search_tr.php.

[12] Polderman, J. W., Willems, J. C., Introduction to Mathematical Systems Theory: A Behavioral Approach, Springer, Berlin, 1997.

[13] Ramadge, P. J., Wonham, W. M., Supervisory Control of a Class of Discrete-Event Processes SIAM Journal of Control and Optimization, Vol. 25, No. 1, pp. 206-230, Jan. 1987

[14] Ramadge, P. J., Wonham, W. M., The control of discrete event systems, Proc. IEEE, vol. 77, no. 1, pp. 81-98, Jan. 1989.

[15] Ruberti, A., Isidori, A., Teoria dei Sistemi, Boringhieri, Torino, Italy, 1979.

[16] Sontag, E. D., Mathematical Control Theory: Deterministic Finite Dimensional Systems. Second Edition, Springer, New York, 1998.

[17] Tomlin, C., Lygeros, J., Sastry, S., Synthesizing Controllers for Nonlinear Hybrid Systems, Lecture Notes in Computer Science, Hybrid Systems: Computation and Control (HSCC98), S. Sastry and T. Henzinger, Eds., vol. 1386, Springer Verlag Berlin Heidelberg, 1998, pp. $360-373$.

[18] van der Schaft, A. J., Schumacher, J. M., Compositionality issues in discrete, continuous, and hybrid systems, International Journal of Robust and Nonlinear Control, vol. 11, pp. 417-434, 2001.

[19] van der Schaft, A. J., Achievable behavior of general systems, Systems \& Control Letters, 49 (2003) pp. 141-149.

[20] van der Schaft, A. J., Equivalence of hybrid dynamical systems, Proc. of Mathematical Theory of Networks and Systems (MTNS 04), Leuven (Belgium), July 5-9, 2004.

[21] van der Schaft, A. J., Equivalence of dynamical systems by bisimulation, IEEE Trans. on Automatic Control, Vol. 49, no. 12, December 2004, pp. 2160-2172.

[22] Willems, J. C., Trentelman, H. L., Synthesis of Dissipative Systems Using Quadratic Differential Forms, Part I, IEEE Trans. on Automatic Control 47 (2002) pp. 53-69. 